\title{
Dancing with Loneliness in Later Life: A Pilot Study Mapping Seasonal Variations
}

\author{
Christina Victor ${ }^{1, *}$, Mary P. Sullivan ${ }^{1}$, Rachel Woodbridge ${ }^{1}$ and Michael Thomas ${ }^{2}$ \\ ${ }^{I}$ College of Health and Clinical Sciences, Brunel University London, Uxbridge, Middlesex UB8 3PH, UK \\ ${ }^{2}$ School of Social Policy, Sociology and Social Research, University of Kent, Gillingham Building, Chatham Maritime, \\ Kent ME4 4AG, UK
}

\begin{abstract}
Temporal variations in loneliness at the individual and population level have long been reported in longitudinal studies. Although the evidence is limited due to methodological distinctions among studies, we broadly know that loneliness as one ages is a dynamic experience with people becoming more or less lonely or staying the same over time. There is, however, less evidence to understand individual variations in loneliness over shorter periods of time. This paper reports on one element of a small mixed method pilot study to investigate seasonal variations in loneliness over the course of one year and to test the effectiveness of tools used to collect data at repeated short intervals. Our findings confirm that loneliness is dynamic even over shorter periods of time with participants reporting to be lonelier in the evenings, weekends and spring-summer period. Data measures were at times problematic due to language and/or interpretation and reinforce the relevance of reviewing the more common approaches to studying loneliness to more effectively capture the complex and individual nature of the experience.
\end{abstract}

Keywords: Loneliness, older people, temporal variations.

\section{INTRODUCTION}

Research reporting the experience of loneliness in later life has a long historical pedigree dating back to the study conducted by Sheldon in Wolverhampton in 1948 [1]. We now have an extensive corpus of work describing the prevalence of loneliness [2], identifying those at risk of experiencing loneliness or key risk factors [3-5], making comparisons across a range of different countries [6] and evaluating interventions to prevent or mitigate loneliness [7]. These studies report the prevalence of loneliness at a population level (e.g. all those aged 65+) at a specific point in time. These types of studies can provide only limited insights into the potentially dynamic nature of the experience of loneliness at a population level. For example, Victor et al. [8] demonstrate the stability of estimates of population level prevalence of loneliness in Britain for those aged 65+ from 1948 to 2014. Using population level data we can also explore variations in loneliness between countries. Stickley et al. [9] described high levels of loneliness in the countries of the former Soviet Union whilst Sundstrom et al. [10] compared loneliness within Europe and reported the "northsouth' divide with levels of loneliness markedly higher for older people in southern countries as compared with northern Europe [11]. Yang and Victor [6] developed this typology further by including both a temporal component by looking at how variations in reported prevalence of loneliness varied

*Address correspondence to this author at the College of Health and Clinical Sciences, Brunel University London, Uxbridge, Middlesex UB8 3PH, UK; Tel: +44 (0)1895 268730; Fax: +44 (0)1895 269853;

E-mail: Christina.victor@brunel.ac.uk between countries and over time. However we know that there are also temporal variations in the experience of loneliness at the individual level. For example, Victor et al. [12] report that when compared to 10 years prior to interview 10 percent of these aged $65+$ reported that they were less lonely and 23 percent that they were more lonely. Crosssectional population level studies of loneliness though cannot clearly articulate how the experience of loneliness may vary over time at both the population and individual level and change in response to the experience of growing old and the challenges that this may generate.

\section{Studying Changes in Loneliness Overtime}

Longitudinal studies offer us the opportunity to investigate how loneliness may (or may not) change over time at both the individual and population level. These types of studies vary in terms of the size and definition of the population, the location of the study, the length of follow up and how loneliness is measured. We can differentiate between studies that focus upon specific localities rather than being nationally based. An exemplar of the former approach is the study based in Tampere, Finland where loneliness for those aged 60+ has been reported at 10 [13] and 28 years follow up [14]. In this study loneliness was measured using a self-report question: 'do you feel lonely' with 'often', 'sometimes', 'never' and 'don't know' as the response categories. These authors reported the 28 year incidence rate for loneliness as $37 \%$. Wenger et al. [15] similarly followed up a population of older people living in North Wales for 25 years. Such locality based studies are valuable but may 
generate spurious findings because of the specific characteristics of the population studied. For example, the study by Wenger et al. [16] reported that speaking Welsh was a 'protective' factor against loneliness, a finding which is unique to the specific rural population studied. Hence the importance of conducting longitudinal studies of ageing that are broadly representative of the national population.

Using national level data Cohen Mansfield et al., Dahlberg et al. (2014) and Victor and Bowling [7, 17, 18] have looked at changes in loneliness over time in nationally based samples of older people in Israel, Sweden and Great Britain respectively. Within this group of studies we can identify two key approaches to studying loneliness longitudinally. One approach as exemplified in the work of by Jylha [13] considers if levels of reported loneliness increase as populations age. This study showed that severe loneliness was 8 percent at both baseline and 10 year follow up whilst Wenger and Burholt [15] reported a modest decrease from 9 to 6 percent over a similar follow up period. Cohen-Mansfield et al. [7] reports that, over a 3.5 year follow up period, mean levels of loneliness increased significantly from 0.62 to 0.8 on a 3 point scale for those aged $60+$ in Israel. However, these studies report overall levels of loneliness for the population as whole meaning that we cannot determine if it is the same (or different) individuals who are reporting loneliness at the two time points.

Complementary to these types of studies, therefore, are those which focus upon enumerating changes in loneliness at the individual level. This approach enables us to determine if it is the same groups of individuals who are reporting loneliness at each follow up point or if this is a dynamic and changing population. Thus a longitudinal approach enables us to differentiate the population who report that they are lonely into 3 distinct groups: those for whom loneliness is a consistent experience and those for whom loneliness may increase/decrease over time $[15,17,18]$. This more nuanced disaggregation into the nature and characteristics of those defined as lonely offers the potential for understanding loneliness across the life course at an individual level. Both cross-sectional and longitudinal studies across a range of different countries and contexts consistently demonstrate that the majority of older people do not experience loneliness in later life. For example, Nummea et al. [19] report that in their follow up study over 3 years that approximately $60 \%$ of older people were never lonely which is identical to the proportion reported by Victor and Bowling [18] over a 10 year follow up period. However, one issue that these longterm longitudinal studies cannot address is how feelings of loneliness may vary over shorter time periods. We have some evidence that feelings of loneliness vary with day of the week and time of day. For example, Bennett and Victor [20] report that, of those who are lonely, it is more common in the evenings and at weekends and during the winter months. This association between winter and presumed increases in loneliness amongst older people is a very redolent image that is used by charities and other interest groups to draw attention to the 'plight' of the lonely old person at Christmas (see the Christmas appeal by Friends of the Elderly: http//www.fote/org.uk).
There is limited empirical evidence that explores variations in loneliness over shorter time periods and supports the commonly held assumption that there are increasing levels of loneliness during the winter months remains limited. To begin to address this gap in our knowledge we undertook a repeated measures pilot survey to investigate both how participants ratings of loneliness vary over the course of a year, as well as loneliness ratings by season, investigating if loneliness rating peak during the winter months. Methodologically we consider the acceptability to participants of completing multiple questionnaires across a comparatively short time interval.

\section{METHODS}

We used mixed methods, consisting of a self-completion postal questionnaire and in-depth interviews, to examine seasonal patterns of loneliness over the course of a 12 month period. Here we report the results of the survey component.

Participants: Our study population was derived from the membership of the university's Older People's Reference Group. This is a volunteer group of older people who support ageing research at Brunel University London by their participating in research, shaping the direction of our research programmes and providing feedback on our findings. All members of the group, approximately 150 in total, were written to and invited to participate. Thirty-four members responded and were enrolled in the study.

Data collection plan: We started our quantitative data collection in the summer of 2012 with subsequent follow-up points linked to the seasons of autumn (September 2012); winter (December 2012); spring (March 2013) and summer (June 2013). We thus collected data at baseline and at 4 follow points providing a maximum of 5 data collection points for each participant.

Measures: We collected a common set of indicators across all data collection waves including: (a) two measures of loneliness - the 6-item de Jong Gierveld scale [21] and a self-report question; (b) for those who answered to the selfreport question the time/day of week they felt lonely; and (c) the season when participants felt loneliness was most problematic. In addition we asked participants to report any significant changes in their lives between data collection points. At baseline we collected standard socio-demographic data (age, sex, marital status, household composition), contact with friends/relatives, health status (self-reported and long term limiting illness and quality of life rating).

Data analysis strategy: Our analysis plan was to: (a) use simple descriptive statistics to report the prevalence of loneliness by season; (b) to classify participants as lonely/not lonely at baseline and track how this changed by season; (c) to examine how reported levels of loneliness were linked with perceptions of the 'loneliest' time of year.

Ethics: Ethical approval was obtained from the Brunel University School of Health and Social Care Research Ethics Committee.

\section{RESULTS}

We present our results in 3 sections linked to our research objectives as stated above. 


\section{PROFILE OF PARTICIPANTS}

Thirty-four participants completed our baseline questionnaire and 28 completed all 5 questionnaires. Reasons for drop out included withdrawal due to the nature of the survey (1), deceased (1 after wave 4), incomplete questionnaires (1) and unknown ( 2 failed to return any questionnaires following reminders). As one participant who initially started the study commented: "Sorry - didn't realise how miserable I'd get doing this. I'd like to withdraw from the study". The prevalence data related to participants who provided data for individual waves $(\mathrm{N}=30)$ whilst the longitudinal results presented in this paper relate to the 28 participants with complete data across all 5 waves plus 1 participant with complete data for waves 1-4. Table 1 demonstrates that the average age of participants was 74 years (range 65-93); 56 percent were female, the majority were home owners $(80 \%)$,

Table 1. Characteristics of participants at baseline $(\mathrm{N}=30)$

\begin{tabular}{|c|c|}
\hline $\mathbf{N}=\mathbf{3 0}$ & Percentage \\
\hline $\begin{array}{l}\text { Age }(y r s) \\
\text { Mean } \\
\text { Range }\end{array}$ & $\begin{array}{l}74.43^{a} \\
65-93\end{array}$ \\
\hline $\begin{array}{l}\text { Gender } \\
\text { Male } \\
\text { Female }\end{array}$ & $\begin{array}{l}43.3 \\
56.7\end{array}$ \\
\hline $\begin{array}{l}\text { Marital Status } \\
\text { Widowed } \\
\text { Married } \\
\text { Divorced/Separated } \\
\text { Single/Never Married }\end{array}$ & $\begin{array}{l}30.0 \\
30.0 \\
20.0 \\
20.0\end{array}$ \\
\hline $\begin{array}{l}\text { Health } \\
\text {-Health Status } \\
\text { Good } \\
\text { Fairly Good } \\
\text { Not Good } \\
\text {-Long standing illness? } \\
\text { That limits activities? }\end{array}$ & $\begin{array}{l}56.7 \\
33.3 \\
10.0 \\
21.0 \\
10.0\end{array}$ \\
\hline $\begin{array}{l}\text { Living } \\
\text {-Accommodation } \\
\text { Owned } \\
\text { Rented } \\
\text { Mortgage/loan } \\
\text {-Length of time in area } \\
+10 \text { years } \\
5-10 \text { years }\end{array}$ & $\begin{array}{c}83.3 \\
10.0 \\
6.7 \\
\\
86.7 \\
13.3\end{array}$ \\
\hline $\begin{array}{l}\text { Wellbeing [28] } \\
\text {-QOL } \\
\text { Very Good } \\
\text { Fairly Good } \\
\text { Neither good nor bad } \\
\text {-Satisfaction with life scale (Diener, Emmons, } \\
\text { Larsen \& Griffin, 1985) } \\
\text { Mean } \\
\text { Range } \\
\text {-General self-efficacy scale [29]Jerusalem \& } \\
\text { Schwarzer, 1981) } \\
\text { Mean } \\
\text { Range }\end{array}$ & $\begin{array}{l}33.3 \\
60.0 \\
6.7 \\
\\
24.48^{\mathrm{b}} \\
12-35\end{array}$ \\
\hline
\end{tabular}

2participants did not report age,

${ }^{\mathrm{b}} 1$ participant did not complete satisfaction with life scale,

2 participants did not complete general self-efficacy scale in good health (both self-rated and in terms of long term limiting illness) and rated their quality of life as good. However, only 30 percent of our participants were married with the rest of our sample being single, widowed or divorced. Given that marital status is a major risk factor for loneliness this may render our sample more vulnerable to loneliness.

\section{THE PREVALENCE OF LONELINESS}

We used two methods of measuring loneliness: the 6 item de Jong Gierveld scale [21] and a self-rating question; 'How often do you feel lonely'. Using the de Jong Gierveld scale in a self-completed questionnaire is unusual as it is typically included in phone or direct interviews. The scale was successfully used in its 11-item format in a self-completion survey in Perth without excessive item non-response [22]. At baseline we could not compute this score for 14 percent of our participants and this increased to 20 percent at wave 5 . In particular there was a systematic pattern of non-completion with participants not completing the 3 negatively worded questions such as 'I miss having people around'. Consequently, we do not include any further analysis of this scale as the responses are unreliable. Thus our study of population and individual levels changes in loneliness over the course of a year are based upon the analysis of our selfreport loneliness question.

At baseline there was no missing data for our question 'How often do you feel lonely?'; and 23.3 percent reported 'never' feeling lonely, 36.7 percent 'rarely' and 40 percent 'sometimes'. No respondent stated they 'often' or 'almost always' feel lonely in this first wave. Given our sample size we examined the face validity of our findings by using our baseline data to look at the relationship between loneliness and key socio-demographic factors. Our aim in undertaking this analysis was to assess the nature of our sample and determine if the pattern of inter-relationships mirrored the existing literature. Age demonstrated a border line negative association with loneliness (i.e. loneliness decreased with age) $(\mathrm{r}=-.365, \mathrm{p}=.056)$ whilst both quality of life $(\mathrm{r}=-.392$, $\mathrm{p}=.032)$ and life satisfaction $(\mathrm{r}=-.593, \mathrm{p}=.001)$ showed significant negative relationships with loneliness. Participants anticipated feelings of loneliness in 10 years was strongly correlated with current ratings of loneliness ( $\mathrm{r}=.501$, $\mathrm{p}=.005$ ), whereas past loneliness was not significantly related $(\mathrm{r}=.131, \mathrm{p}=.490)$. No significant relationships were observed with gender $(\mathrm{r}=-.091, \mathrm{p}=.631)$, household composition ( $)$, health status $(\mathrm{r}=.039, \mathrm{p}=.838)$ or contact with family (brothers/sisters, $\mathrm{r}=-.173, \mathrm{p}=0.454$; children, $\mathrm{r}=$ $-0.218, \mathrm{p}=0.331$; other relatives; $\mathrm{r}=-0.257, \mathrm{p}=.186$ ) and friends $(\mathrm{r}=.052, \mathrm{p}=.790)$. However, there was a negative relationship between loneliness and contact with neighbours $(\mathrm{r}=-.420, \mathrm{p}=.023)$.

Participants who reported that they were lonely 'sometimes', 'often' or 'always' were asked 'are there specific times you feel lonely?' Given the small absolute numbers reporting loneliness at each wave responses were aggregated $(\mathrm{N}=54$, waves $1-5)$ to assess when respondents 
expected to feel lonely. Almost half expected to feel lonely in winter $(46.3 \%)$, compared with 13 percent in summer $(12.96 \%), 11$ percent in autumn $(11.11 \%)$ and 6 percent in spring $(5.56 \%)$. More participants also expected to feel lonely in the evenings (42.59\%) than mornings (14.81\%) and at the weekend (Saturdays, 24.1\%, Sundays, 27.78\%) compared with weekdays.

How do levels of loneliness vary by season for the group overall? Does it mirror the expectations of our participants? The proportion of participants describing themselves as 'never' lonely (aggregating the 'never' and 'rarely' lonely groups) was highest in winter $(68.9 \%)$ and lowest in the initial baseline summer data collection point $(58.6 \%$ ) (see Table 2). Overall the percentage reporting that they were 'never/rarely' lonely is highest in winter and spring and and a further 14 percent had one change in loneliness rating; 17 percent had 2 changes; with the remainder recording 3 (7\%) and 4 (10\%) changes. No significant relationships were observed between changes in loneliness ratings and age, gender, marital status, health or household size.

For each individual we calculated how their loneliness rating changed by season and classified them as a consistent pattern of loneliness, and increasing or decreasing loneliness. To complete this exercise we classified participants as either lonely ('sometimes', 'always', 'often') or not lonely ('never' or 'rarely'). For the 28 participants who completed all waves; 50 percent of responses remained unchanged throughout the year; 10 as not lonely and 4 as lonely. For participants whose loneliness ratings changed, 14.3 percent made one change, 17.9 percent changed twice, 7.1 percent

Table 2. Variations in loneliness

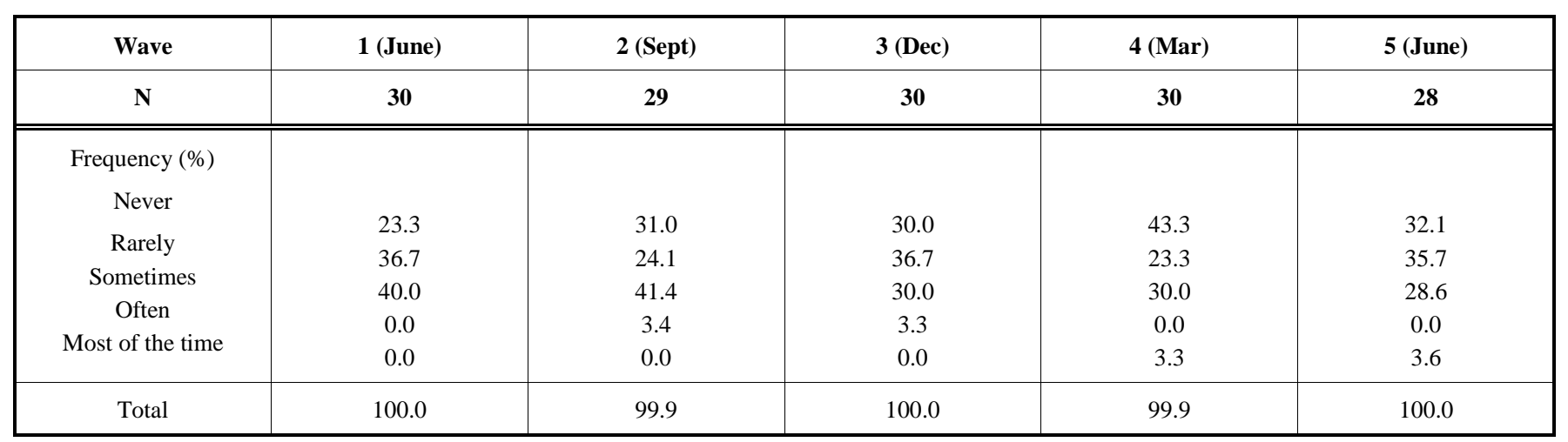

lowest in the summer and autumn. The percentage stating that they were 'often/always' lonely is around 3 percent and shows no obvious seasonally based variations.

How stable are these loneliness ratings for individuals? Whilst Table 2 presents the overall prevalence of loneliness in our sample, it does not tell us about how individuals rated their level of loneliness and how this did (or did not) vary over time. Loneliness ratings were consistent over all 5 waves of data collection for half, 52 percent, of our group changed 3 times and a further 10.7 percent changed every time. Fig. (1) presents these changes graphically for individuals and illustrates the turbulent and dynamic way that loneliness changed for half of our sample over the course of our 12 month follow-up. How did these changes map onto the different times of year? As Table $\mathbf{3}$ shows the season characterised as demonstrating the largest number of individuals with reducing levels of loneliness is the autumnwinter transition whilst the spring-summer transition shows the largest increases in loneliness ratings for our participants.

Table 3. Variations in loneliness by season

\begin{tabular}{|c|c|c|c|c|}
\hline & Decrease in Loneliness & Stayed The Same & Increase in Loneliness & \\
\hline \hline Wave 1-Wave 2 (summer-autumn) & & & & \\
\hline & 5 & 19 & 1 & 29 \\
\hline Wave 2- Wave 3 (autumn to winter) & 9 & 20 & 29 \\
\hline Wave 3-Wave 4(winter to spring) & 5 & 22 & 2 \\
\hline Wave 4-Wave 5(spring to summer) & & & & 29 \\
\hline
\end{tabular}




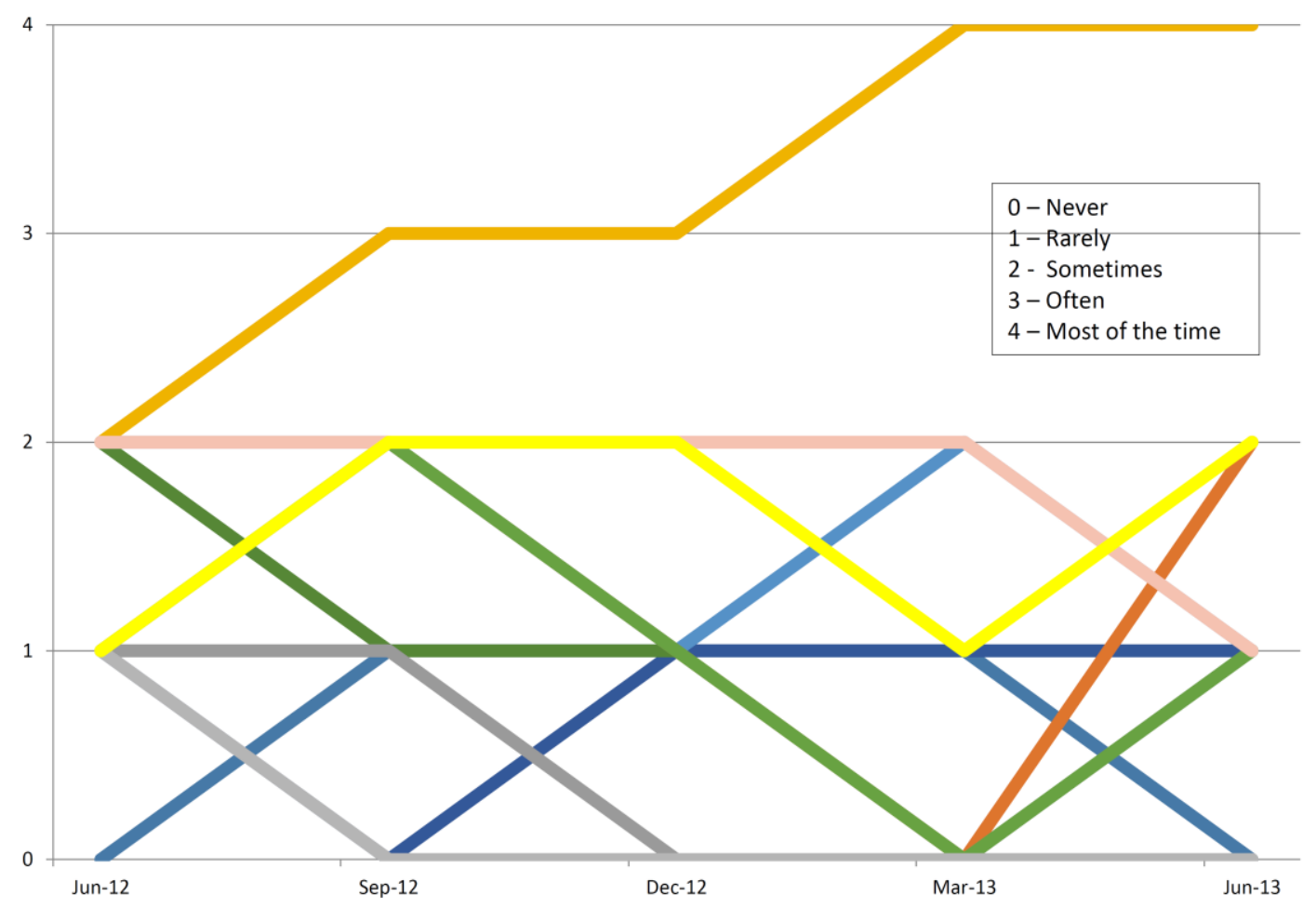

Fig. (1). Variations in loneliness across 12 months.

\section{DISCUSSION}

In this pilot study we sought to address two key questions relating to the study of longitudinal variations in loneliness amongst older people: one methodological and one substantive. Our methodological question focussed upon the acceptability of a year long study of variations in loneliness using a postal questionnaire repeated at comparatively short intervals and the types of measures we included. Our substantive question sought to examine if levels of loneliness vary across the course of a year (i.e. shorter time periods) and, more specifically, do levels of loneliness increase in the winter months as anticipated from previous literature and the emphasis on loneliness in winter by charities working with older people. We discuss each of these questions in turn.

As this was a pilot survey we recruited our participants from our university's Older People's Reference Group. More specifically we wanted participants who could both provide data about the experience of loneliness over the course of a year and engage with us to provide feedback on the experience of being part of the study and how acceptable they thought our approach would be to the wider population. This was explored by both the free text comments provided by participants on the questionnaires and in-depth interviews with 24 participants examining their thoughts on their understanding and experience of loneliness. These interviews will be reported on elsewhere, but we refer to the free text comments for participants reflection on the topic, methods and questions included in the questionnaire.

We measured out main outcome variable, loneliness, using two methods. The self-report question showed very high acceptability to participants with only 1 questionnaire out of 144 with this item missed. However, the de Jong
Gierveld scale [21] was characterised by high levels of item non-response specifically relating to the negatively worded statements. As one participant observed, "Answering 'negative' questions can be confusing". Participants commented upon the 'tick box' response format of the questionnaire and the limiting nature of our response formats: "When I first read through I thought the response ratings were too simplistic. There are ratings I have given that I would have liked to have been better defined" and "I have indicated that I occasionally feel lonely but there are times when I feel very alone which is not exactly the same as lonely". Such issues were not confined to our questions around loneliness but some participants found our taken for granted terms challenging as follows: "I still cannot identify the meaning 'friend"' This example serves to highlight some of the methodological challenges of using a postal questionnaire.

Completing 5 questionnaires across a 12-month period requires significant engagement by participants with the study aims. As one participant observed: "Interesting project and questionnaire that has got me thinking." Of the 34 who initially completed the baseline, 28 completed all 5 data collection points and 29 all 4 suggesting that once participants had completed the first questionnaire they were in some way committed to the study and the completion of further questionnaires. Of the 6 lost to follow up, 1 died between waves 4 and 5, 1 withdrew because they were spending time abroad and 1 because they found the topic of the study upsetting. In planning future studies, we need to mindful that loneliness can be a sensitive topic, and that we must minimise potential distress participants by ensuring that they are fully appraised of the topic of the study, and providing details of agencies that can provide help and 
support. Several participants commented that they would have preferred to complete an online survey and we need to take into account the increasingly on line access of older people.

It is important to acknowledge that this is a sample of volunteers derived from a group of older people interested in ageing research at our university. Inevitably this limits the degree to which we can generalise from our findings. Our response rate of approximately 25 percent to the initial invitation to participate was acceptable given that the profile of our study population approximated to our general population; and levels of reported loneliness approximated to national norms with approximately 3 percent of our group defined themselves as often or 'always' lonely compared with 5 percent for the general population. We do not appear to have recruited a sample of participants who are lonelier/less lonely than their peers despite the high levels of widowhood and divorce. Furthermore the relationships observed between loneliness at baseline and demographic, social contact, health and quality of life ratings are in broadly in line with previous studies. For example, the negative relationship between quality of life and loneliness is well established as are our observation of no relationships with social contact. Thus we can have broad confidence that our study group are not atypical of the general older population. In addition, the 28 individuals who completed all 5 waves generated 140 data collection records so - whilst the sample size may be small in absolute terms - this exercise generated a significant amount of data. Thus we can examine in depth changes in loneliness for individuals over time.

We observed a significant relationship between baseline loneliness ratings and future expectations of increasing levels of loneliness. Expectations about the experience of ageing and later life are highly predictive of quality of life and life satisfaction. We have demonstrated elsewhere [23] that an expectation of old age as a time of loneliness and the anticipation of experiencing loneliness as they grow old are highly predictive of loneliness at follow up for those who were not lonely when they answered these two expectations questions. The link between loneliness and expectations of loneliness are intriguing and merit further research as this quotation from our free text section illustrates: "I feel more lonely than 10 years ago as in that time my husband, other relatives of his and mine and some friends have died. Not unexpected at 79 years old. I don't expect to feel more lonely in 10 years time as I now have younger friends and adult grandchildren". Expecting to become lonely with age may reflect either a realistic evaluation of what old age will be like or the embodiment of ageist negative stereotypes about ageing and the marginalisation and social exclusion of older people.

Our study looked at how loneliness varied across days of the week and times of the day. Victor et al. [22] report that two thirds of those who are lonely experience this at specific times most notably evenings and weekends with women being more likely to report loneliness at weekends than men [20] and this pattern was evident in our data both quantitatively and in the free text comments as follows: "The times of loneliness are late evenings. This is when I miss the companionship of my late husband". Public Holidays (known as Bank Holidays in the UK) were also reported as a focal point for loneliness both quantitatively and in the free text comments "I find bank holiday can be quite lonely" and "Bank holidays can be difficult especially Monday. I work in a charity shop on Monday morning and of course it is not open on Bank Holidays".

Longitudinal studies of loneliness have provided insights into the dynamic nature of loneliness at both population and individual levels of analysis. At the population level longitudinal studies have demonstrated that the group classified as lonely at any specific point in time is constituted of three specific groups: those with a consistent pattern of loneliness; those with increasing loneliness and those with decreasing levels of loneliness. This typology was present in our study indicating that loneliness can vary across the space of 12 months as well as over much longer follow up periods. The different groups constituting the 'lonely' population may go some way to explaining why interventions to prevent/ reduce loneliness have been so unsuccessful [24, 25]. For half of our participants their level of loneliness did not vary over the course of our study. It is difficult to compare this with other longitudinal studies of changes in loneliness as they cover very much longer follow-up periods and the comparisons are probably inappropriate. Rather our study complements existing research by demonstrating that loneliness can vary over the course of a day, week or year as well as much longer periods.

There is an established literature demonstrating elevated levels of depression, suicide and mortality in the winter months in northern Europe [26, 27]. Mirroring this pattern, vulnerability to increased levels of loneliness in winter months has been reported [20]. In particular we wanted to start to examine the received wisdom of our participants, previous research, the general population and charities working with older people that winter generates a peak in loneliness, especially at Christmas time. We examined seasonal variations in loneliness by mapping individual trajectories of loneliness across the year. We looked at changes between waves as we wanted to try to see how fluctuations in loneliness were linked to seasonal variations. Although the numbers are small this was highly complex as each of our participants had 5 measures of loneliness to examine. Unexpectedly given both the literature on seasonal variations in mortality and depression in western and northern Europe and the comments from participants', we see that the most stable period in terms of loneliness rating is the autumn-winter transition. Where changes were observed for this period they were predominantly decreases in loneliness. Conversely the spring-summer period is characterised by the most numerous increases in loneliness. In our study there is no compelling evidence to support the thesis that loneliness ratings increase in the winter months and, more specifically, at Christmas time. Levels of loneliness were not higher in our winter period for the group as a whole and increases in loneliness ratings were not concentrated in this period. This is a finding which merits further investigation in a larger national level study.

However our participants were convinced that loneliness was associated with the winter months. Fifty of those reporting loneliness thought that levels of loneliness were 
highest in the winter months. The observation by one participant that "I think probably winter, everybody will say that" (loneliness is greatest in winter)" was emblematic of our interviewees ideas about seasonal variations in loneliness. What might explain this apparent paradox? Our participants provide some evidence as to why the anticipated spike in loneliness was not evident with comments such as: "I'm spending Christmas with them (children), so yes, that'll be fine". Christmas provides a focal point for families to meet up and spend time together which is, perhaps, lacking in the summer months. Further we may speculate that longer days and the departure of family and friends on holidays may be more problematic for the experience of loneliness than previously anticipated. In addition many activities close down or operate at a reduced level over the summer period because of staff holidays. Clearly these are preliminary findings and they need further investigation by larger studies with more diverse samples. However, these findings also challenge us to address wider stereotypes about loneliness and older people more generally and to develop more insightful and robust interventions that address the times of year when older people experience loneliness and nor when we assume they do.

There are a number of caveats to our findings relating to the small sample size. Inevitably this limited the statistical analysis we were able to undertake precluding the use a regression modelling to examine trends in loneliness by wave. We have demonstrated that this type of intensive study is broadly acceptable to potential participants, that our sample report levels of loneliness at baseline characteristic of the general population and the study has generated novel findings that provide potential avenues for further research. We have demonstrated that loneliness varies over the course of a year at both population and individual level. At the population level our data challenge the common stereotype that loneliness is a 'winter problem'. At the individual level almost half of our participants reported changes in their loneliness ratings ranging from 1-4. This highlights the dynamic and complex nature of loneliness; how it changes with time, both in the short and long term, and its antecedents not always clearly understood. These variations or this dance with loneliness across both short and long periods of time may imply that researching loneliness to capture its true character in later life is more challenging than previously argued. Quantitatively, the nature of the scales that we use and attendant issues of language, or response formats and missing data, limits describing a more nuanced experience. Qualitatively, we similarly overlook changeability or instability in the loneliness experience given the challenges associated with expressing in words its attributes, meanings and consequences so that is easily understood by another. Therefore, if we are to fully understand the experience of loneliness in later life and develop appropriate, acceptable and effective interventions then we need to develop methods that are sufficiently sensitive to reveal the dance with loneliness in later life.

\section{CONFLICT OF INTEREST}

The authors confirms that this article content has no conflict of interest.

\section{ACKNOWLEDGEMENTS}

We wish to express our thanks to those people who participated in the survey. Financial support to undertake this study was provided by Brunel University London.

\section{REFERENCES}

[1] Sheldon JH. The social medicine of old age. London: Oxford University Press 1948.

[2] Dahlberg L, McKee KJ. Correlates of social and emotional loneliness in older people: Evidence from an English community study. Aging Ment Health 2014; 18: 504-14.

[3] Dykstra PA. Older adult loneliness: myths and realities. Eur J Ageing 2009; 6: 91-100.

[4] Pinquart M, Sorenson S. Influences on loneliness in older adults: a meta analysis. Basic Appl Soc Psych 2002; 23: 245-66.

[5] Pikhartova J, Bowling A, Victor CR. Does owning a pet protect older people against loneliness? BMC Geriatr 2014; 14: 106.

[6] Yang K, Victor C. Age and loneliness in 25 european nations. Ageing Society 2011; 31: 1-21.

[7] Cohen-Mansfield J, Shmotkin D, Goldberg S. Loneliness in old age: longitudinal changes and their determinants in an Israeli sample. Int Psychogeriatr 2009; 21: 1160-70.

[8] Victor CR, Scambler SJ, Shah S, et al. Has loneliness amongst older people increased? an investigation into variations between cohorts. Ageing Society 2002; 22: 585-97.

[9] Stickley A, Koyanagi A, Roberts B, et al. Loneliness: Its correlates and association with health behaviours and outcomes in nine countries of the former soviet union. PLoS One 2013; 208: e67978. doi:10.1371/journal.pone.0067978.

[10] Sundstrom G, Fransson E, Malmberg B, Davey A. Loneliness among older Europeans. Eur J Ageing 2009; 6: 267-75.

[11] Fokkema T, De Jong Gierveld J, Dykstra PA. Cross-national differences in older adult loneliness. J Psychol 2012; 146: 201-28.

[12] Victor C, Scambler S, Bond J. The social world of older people, understanding loneliness and social isolation in later life. Maidenhead, Berkshire: Open University Press 2009.

[13] Jylhä, M. Old age and loneliness: Cross-sectional and longitudinal analyses in the tampere longitudinal study on aging. Can J Aging 2004; 23: 157-68.

[14] Van Aartsen M, Jylhä M. Onset of loneliness in older adults: results of a 28 year prospective study. Eur J Ageing 2011; 8: 31-8.

[15] Wenger GC, Davies R, Shahtahmasebi S, Scott A. Social isolation and loneliness in old age: review and model refinement. Ageing Society 1996; 16: 333-58.

[16] Wenger GC, Burholt V. Changes in levels of social isolation and loneliness among older people in a rural area: a twenty-year longitudinal Study. Can J Aging 2004; 23: 115-27.

[17] Dahlberg L, Andersson L, McKee KJ, Lennartsson C. Predictors of loneliness among older women and men in Sweden: A national longitudinal study. Aging Ment Health 2014; 15: 1-9.

[18] Victor CR, Bowling A. A longitudinal analysis of loneliness among older people in Great Britain. J Psychol 2012; 146: 313-31.

[19] Nummela O, Seppänen M, Uutela A. The effect of loneliness and change in loneliness on self-rated health (SRH): A longitudinal study among aging people. Arch Gerontol Geriatr 2011; 52: 33-9.

[20] Bennett K, Victor C. "He wasn't in that chair": What loneliness means to widowed older people. IJAL 2012; 7: 33-52.

[21] De Jong Gierveld J, Van Tilberg T. A 6-item scale for overall, emotional, and social loneliness. Res Aging 2006; 582-98.

[22] Victor CR, Grenade L, Boldy D. Measuring loneliness in later life: a comparison of different measures. Rev Clin Gerontol 2005; 15: 63-70.

[23] Pikhartova J, Bowling A, Victor C. Is loneliness in later life a selffulfilling prophecy? Aging Ment Health 2015; doi: 10.1080/ 13607863.2015.1023767

[24] Windle K, Francis J, Coomber C. Preventing loneliness and social isolation: Interventions and outcomes. Research Briefing 2011; 39.

[25] Cohen-Mansfield J, Perach R. Interventions for alleviating loneliness among older persons: A critical review. Am J Health Promot 2014. doi: dx.doi.org/10.4278/ajhp.130418-

[26] Hajat S, Barnard LT. Cold-related mortality and morbidity. In: R. A. Pielke, Ed. Climate Vulnerability. Oxford: Academic Press 2013; pp. 157-64. 
[27] Kasof J. Cultural variation in seasonal depression: cross-national differences in winter versus summer patterns of seasonal affective disorder. J Affect Disord 2009; 115: 79-86.

[28] Diener E, Emmons RA, Larsen RJ, Griffin S. The satisfaction with life scale. J Pers Assess 1985; 49: 71-5.
[29] Jerusalem M, Schwarzer R. Self-efficacy as a resource factor in stress appraisal. In R. Schwarzer, Ed. Self-efficacy: thought control of action. Washington, DC: Hemisphere 1992; pp. 195-216.

Received: October 25, 2014

Revised: January 30, 2015

Accepted: February 02, 2015

(C) Victor et al.; Licensee Bentham Open.

This is an open access article licensed under the terms of the Creative Commons Attribution Non-Commercial License (http://creativecommons.org/licenses/by-nc/3.0/) which permits unrestricted, non-commercial use, distribution and reproduction in any medium, provided the work is properly cited. 